\title{
Closed-loop ground layer adaptive optics simulations with elongated spots : impact of modeling noise correlations
}

\author{
Clémentine Béchet ${ }^{1, \text { a }}$, Miska Le Louarn ${ }^{1}$, Richard Clare ${ }^{1}$, Michel Tallon ${ }^{2}$, Isabelle Tallon-Bosc ${ }^{2}$, and \\ Éric Thiébaut ${ }^{2}$ \\ 1 European Southern Observatory (ESO), Karl-Schwarzschild-Strasse 2, 85748 Garching, GERMANY \\ 2 Centre de Recherche Astrophysique de Lyon - Observatoire de Lyon, 9 Avenue Charles André 69561 \\ Saint Genis Laval cedex, FRANCE
}

\begin{abstract}
Laser guide stars on an Extremely Large Telescope (ELT) produce elongated spots on the subapertures of the Shack-Hartmann wavefront sensors for adaptive optics (AO) correction. This degrades the measurement accuracy and introduces structured correlations in the centroiding errors, depending on the position of the laser launch telescope with respect to the sub-aperture location inside the pupil. Advanced centroiding algorithms are used to improve the measurement accuracy, but closed-loop control methods in adaptive optics usually neglect the structured error correlations. However, properly modeling the noise correlations is expected to improve the correction. We take into account structured errors statistics inside a fast algorithm for wavefront reconstruction and control on a 42-meter telescope. The closed-loop performance of the new algorithm, including a maximum a posteriori reconstruction and a pseudo open-loop control with an integrator is compared to the uniform and uncorrelated noise model used in classical AO corrections. End-to-end simulations are used to compare the two approaches. A ground layer AO configuration is simulated. The results provide additional information to the discussion about the choice of laser launch telescope positions on an ELT-class telescope.
\end{abstract}

\section{Introduction}

Laser guide stars (LGS) are supposed to open the way for adaptive optics in fields containing only faint stars [1] but their use on Extremely Large Telescopes (ELT) is more complicated than on existing 10-meter class telescopes. We investigate here one of the reasons for this, which is the large spot elongation produced on the Shack-Hartmann wavefront sensors (WFS), degrading the measurement quality. At a first stage, advanced algorithms for centroiding [2] have been tested with classical reconstruction and control to mitigate this effect. Structured correlations in the measurements noise have also been modeled [3] in a multi-LGS configuration showing a significant improvement of wavefront reconstruction quality.

In this paper, we demonstrate the benefit of an improved noise model in the reconstructor for a Ground Layer Adaptive Optics (GLAO) configuration, using end-to-end simulations on a 42-m telescope. First, the simulated GLAO configuration and the reconstruction and control strategy are described. In Sect. 3, the improved model of the noise involved in the reconstruction is detailed. Then, in Sect. 4, simulations results are presented and the benefit of the improved noise model is discussed.

\section{GLAO system description}

We have used the ESO simulator for large AO systems [4] to simulate a GLAO on a 42-meter telescope. An atmosphere of ten turbulent layers is simulated, following von Kármán statistics with an outer scale $L_{0}=25 \mathrm{~m}$. Wind speeds and $C_{n}^{2}$ profiles values were extracted from the baseline values for EuropeanELT studies, deduced from measurements campaigns at Cerro Paranal [5]. Three typical profiles have been used, referred hereafter as good, median and bad turbulence respectively. The relative vertical distribution of the $C_{n}^{2}$ for the three profiles is similar but the seeing values are respectively $0.6,0.8$ and

\footnotetext{
a e-mail: cbechet@eso.org
}

This is an Open Access article distributed under the terms of the Creative Commons Attribution-Noncommercial License, which permits unrestricted use, distribution, and reproduction in any noncommercial medium, provided the original work is properly cited. 
1.1 arc-seconds for the good, median and bad profiles. Since the $C_{n}^{2}$ distribution has a strong influence on GLAO performance, it is important to mention that in each of these simulated atmospheres there is approximately $50 \%$ of the turbulence located below $100 \mathrm{~m}, 60 \%$ below $400 \mathrm{~m}$ and $70 \%$ below $800 \mathrm{~m}$.

The Ground layer (GL) is probed by 4 LGS equispaced on a circle of 5 arc-min of diameter on the sky. There is one Shack-Hartmann WFS per guide star. The 4 WFS are identical, with 84 subapertures across the pupil diameter. Optical wave propagation among the atmosphere and the WFS optical system are simulated, which leads to spot images on the pixels of the detectors in each WFS. A centroiding algorithm detailed in Sect. 3 is used to get measurements of the gradients of the wavefront coming from each LGS. In order to focus on the spot elongation issue, the tip-tilt undetermination associated to LGS is not simulated here. The LGS thus allow to measure the tip-tilt and the GLAO system works with only 4 LGS, without any additional natural guide star (NGS).

For the reconstruction of the ground layer, we first model the wavefront sensing as a linear process:

$$
\boldsymbol{d}_{k}=\mathbf{S}_{k} \cdot \boldsymbol{w}_{k}+\boldsymbol{e}_{k} \quad \text { for } \quad k=1 \text { to } 4 .
$$

where $k$ is the index of the WFS, $\boldsymbol{d}$ is the gradients vector, $\mathbf{S}$ is the linear model of the sensing process, $\boldsymbol{w}$ is the turbulent wavefront analyzed by the WFS and $\boldsymbol{e}$ is the noise vector on the measurements. We approximate the sensing process by considering that a ground layer of the atmosphere $w^{\mathrm{GL}}$ is identically observed by all WFS. This layer being close to the ground it is not affected by anisoplanatic effects in the various LGS directions. And the rest of the turbulence, due to higher layers, is considered to have an additional stochastic contribution to measurements denoted $\boldsymbol{d}_{k}^{\mathrm{HL}}$, specific to each guide star direction. The measurement equation (1) can thus be replaced by

$$
\boldsymbol{d}_{k}=\mathbf{S} \cdot \boldsymbol{w}^{\mathrm{GL}}+\boldsymbol{d}_{k}^{\mathrm{HL}}+\boldsymbol{e}_{k} \quad \text { for } \quad k=1 \text { to } 4 .
$$

The principle of GLAO is to reject the contribution of the high-altitude layers which are decorrelated from one LGS direction to another, in order to correct only for the common distortions $w^{\mathrm{GL}}$. However, the terms $\boldsymbol{d}_{k}^{\mathrm{HL}}$ are not exactly uncorrelated from one star to the other because of the statistics of the turbulence in a given layer. At smaller distances than the outer scale of the spatial coherence of the turbulence, the phase distortions are correlated. Here, we consider the terms $\boldsymbol{d}_{k}^{\mathrm{HL}}$ as an additional error contribution, so the approximate model for the measurements becomes

$$
\boldsymbol{d}_{k}=\mathbf{S} \cdot \boldsymbol{w}^{\mathrm{GL}}+\boldsymbol{n}_{k} \quad \text { for } \quad k=1 \text { to } 4
$$

where $\boldsymbol{n}_{k}=\boldsymbol{d}_{k}^{\mathrm{HL}}+\boldsymbol{e}_{k}$ accounts for all discrepancies between model and data, that is to say measurement noise but also model approximations like $\boldsymbol{d}_{k}^{\mathrm{HL}}$ for instance. The GL correction can benefit from a good modeling of statistics of both contributions $\boldsymbol{d}_{k}^{\mathrm{HL}}$ and $\boldsymbol{e}_{k}$. In this paper, an optimized model for the errors $\boldsymbol{e}_{k}$ on spot elongation centroiding is investigated, while the other error contribution coming from high altitude layers is neglected. Therefore, we assume $\boldsymbol{n}_{k} \simeq \boldsymbol{e}_{k}$, with zero-mean Gaussian statistics and no correlations between wavefront sensors.

The GL is estimated from maximum a posteriori reconstruction, determining the solution $\widehat{w^{\mathrm{GL}}}$ of the following linear system [3]

$$
\left(\mathbf{S}^{\mathrm{T}} \cdot\left(\sum_{k=1}^{4} \mathbf{C}_{k}^{-1}\right) \cdot \mathbf{S}+\mathbf{C}_{w \mathrm{GL}}^{-1}\right) \cdot \widehat{\boldsymbol{w}^{\mathrm{GL}}}=\mathbf{S}^{\mathrm{T}} \cdot\left(\sum_{k=1}^{4} \mathbf{C}_{k}^{-1} \cdot \boldsymbol{d}_{k}\right)
$$

where $\mathbf{C}_{k}=\left\langle\boldsymbol{n} \cdot \boldsymbol{n}^{\mathrm{T}}\right\rangle$ is the noise covariance matrix associated to the $k-$ th WFS, and $\mathbf{C}_{\boldsymbol{w G L}}^{-1}$ is the inverse covariance matrix of the wavefront in the GL. The latter is modeled thanks to the fractal approximation of von Kármán turbulence statistics, described in Béchet et al. [6]. The noise model used to build $\mathbf{C}_{k}^{-1}$ is detailed in Sect. 3.

The GLAO system is simulated in closed-loop. A modified pseudo open-loop control [7] is applied with the MAP reconstruction computed by the Fractal Iterative Method [8]. Next, from the estimated GL wavefront, a minimum-norm maximum likelihood fit of this wavefront by the deformable mirror influence functions is computed, leading to a command vector. Finally, an integrator is used for the control in the pseudo open-loop control scheme. 


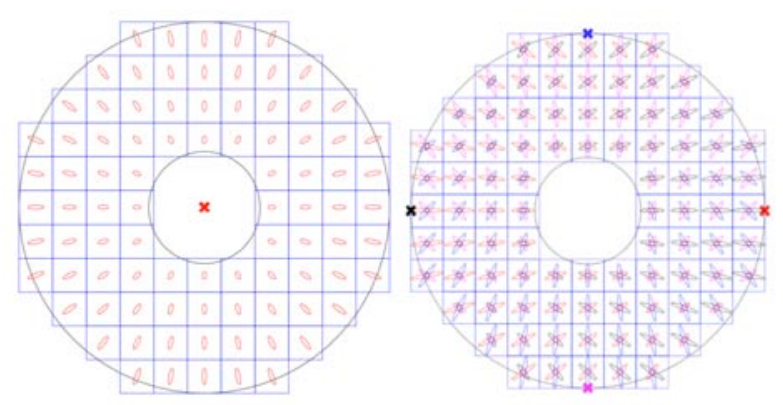

Fig. 1. Layout of the elongated spots among the sub-apertures for 4 LGS launched from the crosses. On the left : Central launch. On the right : Side launch

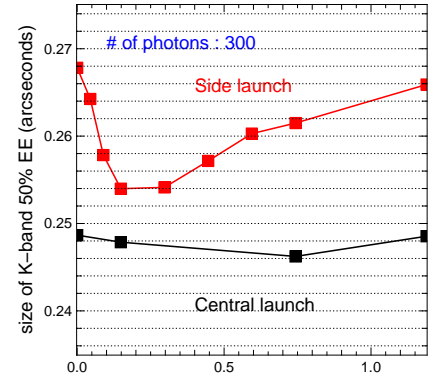

used fraction of elongation prior

Fig. 2. Linear size of the square box with $50 \%$ energy $v s$ fraction of elongation prior, $\alpha$. Black : Central launch. Red : Side launch.

\section{Noise model with elongated spots}

The 4 LGS are generated by the back-scattered light from the sodium layer in the atmosphere, simulated at a central altitude $h_{0}=90 \mathrm{~km}$ and with a Gaussian vertical density profile of full width at half maximum $\mathrm{FWHM}_{\mathrm{Na}}=11.4 \mathrm{~km}$. Due to the vertical extension of the sodium layer, the LGS is seen as an elongated source from sub-apertures located far from the launching point of the laser in the pupil plane. The elongation of the FWHM of the spot intensity in 2 dimensions is approximately given by the elongation vector [3]

$$
\beta \simeq \frac{\mathrm{FWHM}_{\mathrm{Na}}}{h_{0}^{2}}\left(\boldsymbol{x}_{b}-\boldsymbol{x}_{\mathrm{LLT}}\right)
$$

where vectors $\boldsymbol{x}_{b}$ and $\boldsymbol{x}_{\mathrm{LLT}}$ are respectively the coordinates of the center of the sub-aperture and the laser launch telescope (LLT) in the pupil plane. For a 42-m telescope, when the LLT is located behind the secondary, the FWHM of the spot in the most elongated direction is $\beta_{\max }=6$ " in sub-apertures on the edge of the pupil. For a side launching, that is when the LLT's are on the edge of the primary mirror, the most elongated spots will have a FWHM twice as big, that is to say $\beta_{\max }=12$ ". Those two configurations are illustrated at a reduced scale in Fig. 1, showing the layout of the spot elongations over the pupil. In our simulations, behind each sub-aperture is a 20x20-pixel detector, with a maximum field of view of 19.8". Read-out noise of $3 e-$ per frame and per pixel is simulated too.

Thanks to a centroiding algorithm applied on the spots, photo-center displacements (referred as slopes) come out of the WFS's. The elongation strongly degrades the accuracy of the classical algorithm computing center of gravity of the spot image. Advanced centroiding algorithms have been designed to minimize the error on the displacement estimate in case of elongated spots [9][10]. Here, the matched filter [9] is used with a reference intensity deduced from the theoretical parameters of the sodium layer and of the WFS.

The spot elongation affects the statistics of errors $\boldsymbol{e}$ on measurements. With a NGS, the error can usually be considered as uniform and uncorrelated, so the noise covariance matrix $\mathbf{C}_{k}$ for data from the $k$-th WFS is written $\mathbf{C}_{k}=\sigma_{\boldsymbol{e}}^{2} \mathbf{I}$, where $\mathbf{I}$ is the Identity matrix and $\sigma_{\boldsymbol{e}}^{2}$ is the noise variance. With elongated spots, the noise variance is larger in the elongated direction, and in each sub-aperture the displacements in $\mathrm{X}$ and $\mathrm{Y}$ have correlated noises as soon as the elongation is not collinear to $\mathrm{X}$ nor Y. The noise components of $\boldsymbol{e}$ in Eq. (1) from different sub-apertures and also from different WFS's remain however independent. The noise covariance $\mathbf{C}_{k}$ is then a $2 \times 2$ block diagonal (one block per sub-aperture). Considering photon noise only, Tallon et al. [3] write the $2 \times 2$ blocks as

$$
\left[\mathbf{C}_{k}\right]_{2 \times 2}=\sigma_{\boldsymbol{e}}^{2}\left(\begin{array}{cc}
1+\beta_{1}^{2} / \sigma^{2} & \beta_{1} \beta_{2} / \sigma^{2} \\
\beta_{1} \beta_{2} / \sigma^{2} & 1+\beta_{2}^{2} / \sigma^{2}
\end{array}\right),
$$

where $\beta_{1}$ and $\beta_{2}$ are the components of the elongation vector $\beta$ (and $\beta^{2}=\beta_{1}^{2}+\beta_{2}^{2}$ ) and $\sigma$ is the FWHM of the non-elongated spot. Detector pixels, finite FOV, read-out noise, simulated here in the WFS 
modify the statistics of the noise. However, we still approximate the noise by a Gaussian stochastic process with an error depending on the elongation. We write the noise covariance with blocks modeled as follows

$$
\left[\mathbf{C}_{k}\right]_{2 \times 2}=\sigma_{\boldsymbol{e}}^{2}\left(\begin{array}{cc}
1+\alpha^{2} \beta_{1}^{2} / \sigma_{\mathrm{PSF}}^{2} & \alpha^{2} \beta_{1} \beta_{2} / \sigma_{\mathrm{PSF}}^{2} \\
\alpha^{2} \beta_{1} \beta_{2} / \sigma_{\mathrm{PSF}}^{2} & 1+\alpha^{2} \beta_{2}^{2} / \sigma_{\mathrm{PSF}}^{2}
\end{array}\right),
$$

where the scalar $\alpha$ allows to tune the relative increase of the noise with respect to the elongation and the FWHM of the lens-let point spread function (PSF) is denoted $\sigma_{\mathrm{PSF}}$. A complete study of the noise level produced by matched filter algorithm would be necessary to theoretically determine the best $\alpha$.

From this structured noise model for spot elongation in Eq. (7), the inverse noise covariance matrix can be easily deduced and it is also $2 \times 2$-block diagonal, with each block corresponding to

$$
\left[\mathbf{C}_{k}\right]_{2 \times 2}^{-1}=\frac{1}{\sigma_{e}^{2}} \frac{\sigma_{\mathrm{PSF}}^{2}}{\sigma_{\mathrm{PSF}}^{2}+\alpha^{2} \beta^{2}}\left(\begin{array}{cc}
1+\alpha^{2} \beta_{2}^{2} / \sigma_{\mathrm{PSF}}^{2} & -\alpha^{2} \beta_{1} \beta_{2} / \sigma_{\mathrm{PSF}}^{2} \\
-\alpha^{2} \beta_{1} \beta_{2} / \sigma_{\mathrm{PSF}}^{2} & 1+\alpha^{2} \beta_{1}^{2} / \sigma_{\mathrm{PSF}}^{2}
\end{array}\right) .
$$

One should also notice that when $\alpha$ is set to zero, the inverse covariance expression in Eq. (8) is reduced to the inverse covariance for a uniform and decorrelated noise model, as for NGS, $\mathbf{C}_{k}^{-1}=1 / \sigma_{e}^{2} \mathbf{I}$.

This noise model is used in the MAP reconstructor of the closed-loop control (cf. end of Sect. 2).

\section{Simulations results}

The objective of our simulations is to compare the influence of the optimized noise model on the LGS GLAO system performance. The GLAO must improve the energy concentration in the image compared to atmospheric seeing-limited conditions, over a wide field of view. This performance is quantified here thanks to the size of the 50\% ensquared energy (EE) in arc-seconds computed looking at an unresolved on-axis source during 400 milliseconds.

The graph of Fig. 2 shows the performance as a function of the $\alpha$ value, for an average flux of 300 photons per sub-aperture per frame. As mentioned in Sect. 3, $\alpha=0$ means that we assumed a uniform and decorrelated noise model, like in classical reconstructors. There is a minimum size of $50 \% \mathrm{EE}$ for an optimal value $\alpha$ different from zero. The minimum is obtained for approximately $\alpha=0.7$ for central launch (black), and closer to 0.2 for side launch (red). A reduction of $\sim 0.015$ " of the size of the box of 50\% EE is obtained when the improved noise model is used for side launch. A smaller benefit is noticed for central launch. In the following, for every simulation, non-optimized $(\alpha=0)$ and optimized noise models are compared.

Figure 3 presents the two laser launching strategies separately with respect to the LGS flux: central launch (left) and side launch (right). The blue line illustrates the performance reached if one does not correct for any turbulence. At high flux for all the curves, the size of the 50\% EE box is $\sim 4$ times smaller in area than with the atmospheric seeing conditions. In this regime, the measurement errors become negligible compared to the other sources of noise in the GLAO system. When reaching lower flux than 100 photons per frame per sub-aperture, the central launch configuration starts to benefit from the optimized model noise (solid black curve), but the improvement is small. Below 300 photons per frame per sub-aperture, the performance of the side launch configuration with non-optimized noise model (dashed red curve) is dramatically degraded. At very low flux, it does even worse than not correcting at all. However, optimizing $\alpha$ (red solid curve) strongly improved the performance at low flux.

The same results are presented in another way in Fig. 4, comparing on each graph, central (black) and side (red) launch of the lasers. When the noise model is not optimized, the side launch configuration is penalized at flux level below 300 photons. This is due to elongations that are two times larger than for central launch. However, as soon as $\alpha$ is optimized, taking into account the non uniform noise level and the noise correlations, central and side launch provide almost the same performance (graph on the right). This result extends the conclusions of Tallon et al. [3] to closed-loop GLAO. Side launch takes benefit of the varying spot geometries ( $c f$. Fig. 1) to provide the same reconstruction quality as central launch does. 
Clémentine Béchet et al.: GLAO simulations with elongated spots : modeled noise correlations
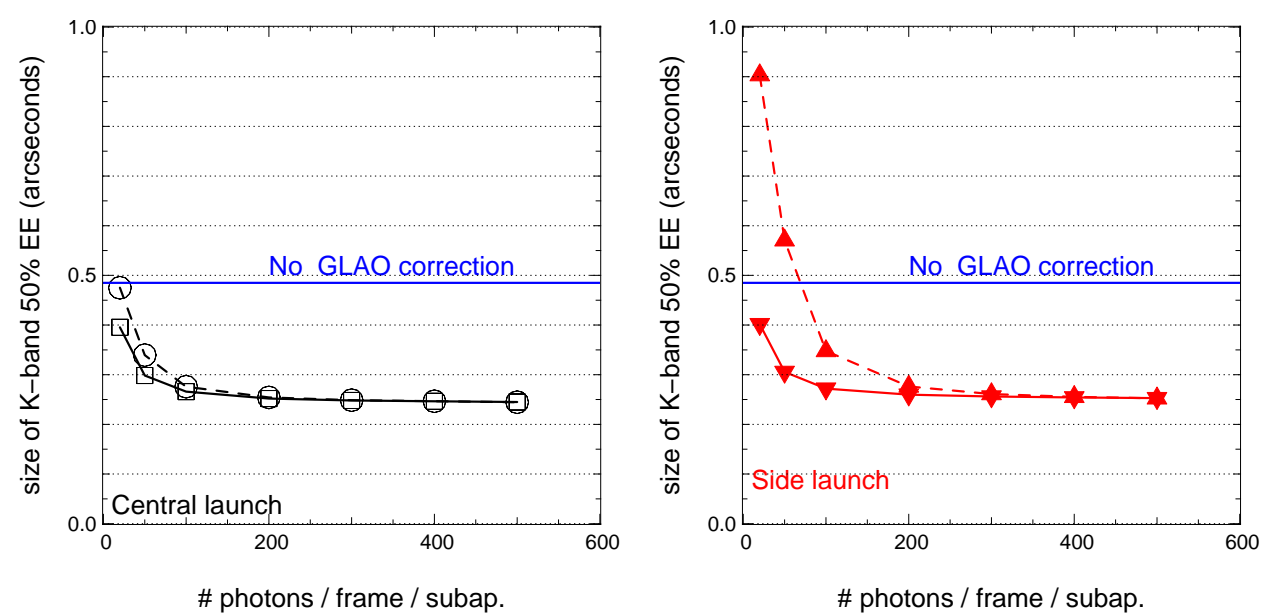

Fig. 3. Linear size of the square box of 50\% energy $v s$ the average number of photons per frame and per subaperture. Dashed : Uniform and decorrelated noise model. Solid : Non uniform and correlated noise model. On the left : Central launch. On the right : Side launch.
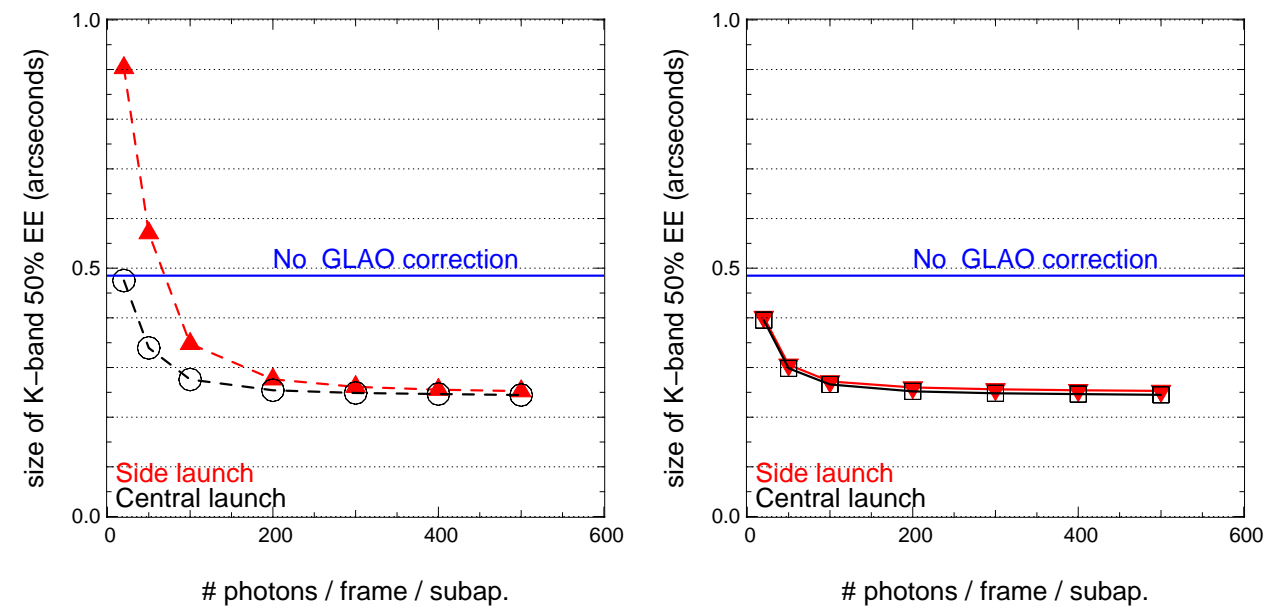

Fig. 4. Same results as in Fig. 3 but central launch (black) and side launch (red) are presented together. On the left : Uniform and decorrelated noise model. On the right : Non-uniform and correlated noise model.

The largest spots for side launch are truncated because of the small detector size, without noticeable degradation of the correction. However, it is worth mentioning that the effect of the truncation on side launch performance may be underestimated here, because we simulate a median, static and symmetric Gaussian sodium density profile [11].

While Figure 3 is obtained with the median atmospheric profile, the simulations are repeated with the good and the bad profiles (See Fig. 5). The general behavior is identical to the previous simulations, just translated toward better or worse absolute performance level for good and bad profiles respectively. Again on the left with a classical noise model in the reconstructor the side launch configuration is penalized for flux below 300 photons. The noise model optimization (right) allows to have the same performance for central and side launch at every flux.

These results demonstrate that the LGS GLAO performance is not degraded by launching from the side of the telescope, as soon as the non-uniform and correlated noise model is used. Also, side launch may be easier to carry out than launching from behind the secondary. For a given laser power, simplified beam relays could then produce more flux for side launch than for central launch. In such case, side launch would then provide better performance than central launch according to results in 
First conference on Adaptive Optics for Extremely Large Telescopes
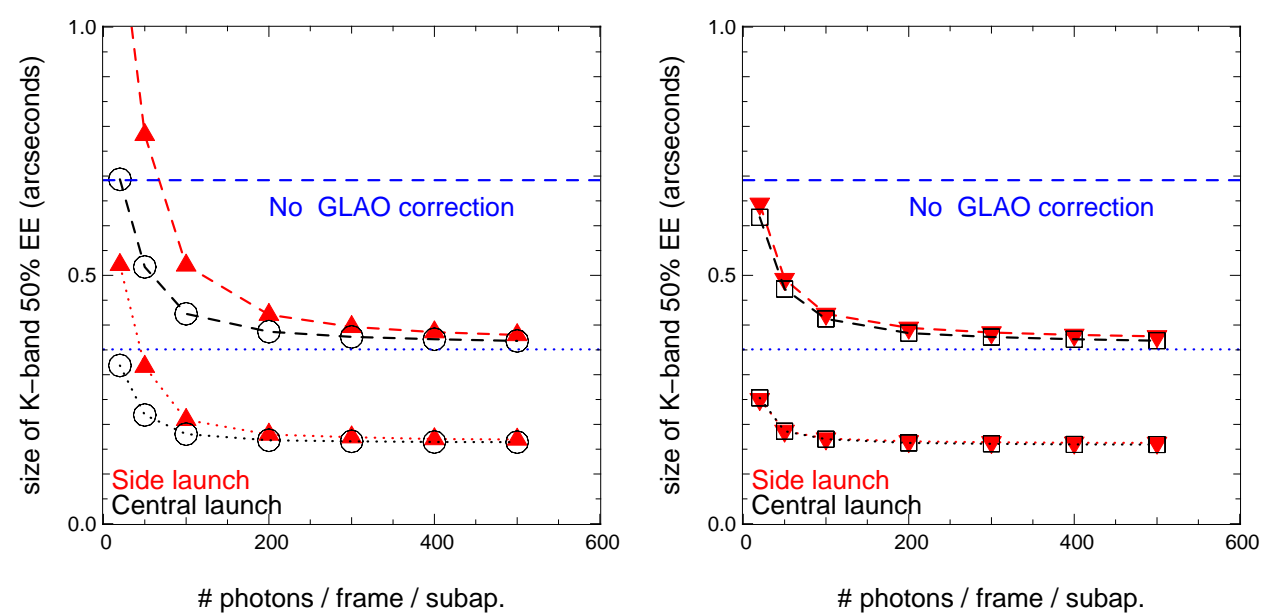

Fig. 5. Same legend as for Figure 4, but for two different atmospheric profiles. Dashed curves and line : bad profile. Dotted curves and lines : good profile.

Figs. 4 and 5. In addition to this, the fratricide effect, not included in our study, is expected to degrade the central launch performance. Further investigation about the LLT scheme and optics is required to determine a quantitative superiority of the side launch.

\section{Conclusion}

Spot elongation was considered a major concern for the choice of the laser launch telescopes location for ELTs because classical noise model in the reconstructor shows a strong disadvantage of side launch configuration. We have presented the first end-to-end simulations of a closed-loop AO system on an ELT-class telescope taking into account the structured noise covariance due to elongated spots in the reconstructor. Thanks to this improved processing method, side launch and central launch configurations lead to the same level of performance, whatever the LGS flux and whatever the turbulence strength.

\section{References}

1. R. Foy and A. Labeyrie, Astron. Astrophys. 152, (1985) 29

2. R. Clare and M. Le Louarn, AO4ELT (2009)

3. M. Tallon, I. Tallon-Bosc, C. Béchet and É. Thiébaut, Adaptive Optics Systems: Proceedings of SPIE 7015, (2008) 70151N

4. M. Le Louarn, C. Vérinaud, V. Korkiakoski, and E. Fedrigo, Advancements in Adaptive Optics: Proceedings of SPIE 5490, (2004) 705

5. S. Oberti, E-ELT AO design inputs: relevant atmospheric parameters (ESO Technical Report, Germany, 2008)

6. C. Béchet, M. Tallon, É. Thiébaut, JOSA A. 26, (2009) 497

7. L. Gilles, Applied Optics 44, (2005) 993

8. M. Tallon, E. Thiébaut and C. Béchet, Adaptive Optics: Analysis and Methods: Proceedings of OSA Conference (2007) PMA2

9. L. Gilles, and B.L. Ellerbroek, Applied Optics 45, (2006) 6568

10. S. Thomas, T. Fusco, A. Tokovinin, M. Nicolle, V. Michau and G. Rousset, MNRAS, 371, (2006) 323

11. R. M. Clare, M. A. van Dam and A. H. Bouchez, Optics Express 15, (2007) 4711 